\title{
FATORES ASSOCIADOS À AFILIAÇÃO RELIGIOSA EM ADOLESCENTES DO ENSINO MÉDIO
}

\section{FACTORS ASSOCIATED WITH RELIGIOUS AFFILIATION IN HIGH SCHOOL TEENAGERS}

\author{
Claudio Bispo de Almeida ${ }^{1}$, Luciene Dias Bispo Veiga², Franck Nei Monteiro Barbosa², Cezar \\ Augusto Casotti ${ }^{2}$ \\ Universidade do Estado da Bahia - UNEB ${ }^{1}$, Universidade Estadual do Sudoeste da Bahia - UESB ${ }^{2}$
}

\begin{abstract}
This study aimed to estimate the prevalence and sociodemographic and behavioral factors associated with religious affiliation in high school students in the municipality of Jequié, Bahia, Brazil. This is a cross-sectional, school-based epidemiological study with a simple random sample of adolescents. A questionnaire applied in the teaching units was used to obtain the data, and later tabulated in duplicate in the Epi DATA program, and after corrections were analyzed in the SPSS version 21.0 program. Initially, a descriptive analysis of the variables was performed, and then the logistic regression analysis was performed. The study included 840 adolescents, predominantly: females (60\%); from the age group of 17 to 19 years (51\%); with adequate consumption of fried foods (63.9\%), fruits (73.3\%) and vegetables (66.2\%), and with inadequate consumption of sweets (59\%); physically inactive (55.7\%); and with religious affiliation (84.6\%). Female adolescents (OR $=2.28$; 1.52-3.40) and male adolescents who were children of mothers with a better level of education (OR = 1.93; 1.10-3.38) were more likely to have religious affiliation, and male adolescents with a higher age group (OR $=0.52 ; 0.29-0.92$ ) were less likely to have any religious affiliation. Thus, it is concluded that the outcome was associated with sociodemographic variables (gender, age group and educational level of mothers).
\end{abstract}

Key words: Adolescent Health, Religion, Life Style, Feeding Behavior, Exercise.

\section{Resumo}

O presente estudo teve o objetivo de estimar a prevalência e fatores sociodemográficos $e$ comportamentais associados à afiliação religiosa em escolares do ensino médio no município de Jequié, Bahia, Brasil. Trata-se de estudo epidemiológico transversal, de base escolar, com amostra aleatória simples de adolescentes. Utilizou-se para obtenção dos dados um questionário aplicado nas unidades de ensino, $e$ posteriormente tabulado em duplicata no programa Epi DATA, e após correções foram analisados no programa SPSS versão 21.0. Inicialmente realizou-se análise descritiva das variáveis, e em seguida fez-se a análise a regressão logística. Participaram do estudo 840 adolescentes, predominando: os do sexo feminino (60\%); do grupo etário de 17 a 19 anos (51\%); com consumo adequado de frituras (63,9\%), frutas $(73,3 \%)$ e verduras $(66,2 \%)$, e com consumo inadequado de doces (59\%); inativos fisicamente (55,7\%); e com afiliação religiosa $(84,6 \%)$. Adolescentes do sexo feminino $(O R=2,28$; 1,52-3,40) e aqueles do sexo masculino filhos de mães com melhor nível de escolaridade (OR=1,93; 1,10- 3,38) tiveram mais chances de possuir afiliação religiosa, e os adolescentes do sexo masculino com maior faixa etária $(O R=0,52 ; 0,29$ 0,92) tiveram menor chance de ter alguma afiliação religiosa. Assim, conclui-se que o desfecho foi associado às variáveis sociodemográficas (sexo, grupo etário e nível de escolaridade das mães).

Palavras chave: Saúde do Adolescente, Religião, Estilo de Vida, Comportamento Alimentar, Exercício. 


\section{Introdução}

Os adolescentes, em geral, se apresentam expostos a comportamentos de risco à saúde, como por exemplo, o consumo de álcoo ${ }^{1-3}$, tabaco $^{3-5}$, baixos níveis de atividade física ${ }^{3}$, hábitos alimentares inadequados (baixa ingestão de saladas, hortaliças, legumes e frutas, alimentação rica em gorduras, açúcares e sal) ${ }^{6,7}$, dentre outros. Tais comportamentos podem ser caracterizados como integrantes do estilo de vida, o qual pode ser entendido como o conjunto de ações habituais que refletem as atitudes, os valores e as oportunidades na vida das pessoas, e pode abranger aspectos positivos ou negativos à saúde ${ }^{8}$.

A prática religiosa tem sido associada ao estilo de vida de adolescentes, seja de forma negativa em escolares de Pelotas-RS ${ }^{1}$, ou positiva nos adolescentes da rede estadual de Pernambuco ${ }^{9}$. Estudo com esta mesma população cita a religião como mediadora para a adesão à comportamentos benéficos à saúde, tendo em vista que as práticas religiosas possuem doutrinas que adotam aconselhamentos positivos na vida dos adolescentes, contribui na formação de valores e práticas saudáveis entre aqueles que aderem a uma religião. Nesta fase da vida a convivência em grupo pode levar à aquisição de comportamentos próprios da idade ${ }^{10}$.

Outro estudo avaliou o estilo de vida de adolescentes de Pelotas-RS, e identificou associação negativa entre ter recebido instrução religiosa e ingestão de álcool nos últimos 30 dias; e entre tempo de entretenimento em programas de televisão maior que duas horas com tempo de prática religiosa maior de 10 anos $^{1}$. Entretanto, em outros grupos de adolescentes, percebe-se que a religiosidade, independente da religião está associada à proteção aos comportamentos de risco à saúde (consumir álcool, cigarros, drogas ilícitas e ter um número elevado de parceiros sexuais) quando comparados aos seus pares que relataram não possuir religião ${ }^{11}$.

Apresentar um estilo de vida negativo, por meio da manutenção de comportamentos de risco modificáveis, pode ser o fator de risco mais prevalente para o desenvolvimento de doenças crônicas não transmissíveis em adolescentes. Portanto, a identificação precoce de comportamentos nocivos à saúde pode possibilitar mudanças nesses comportamentos ${ }^{12}$. Acredita-se que o suporte oferecido pelas instituições religiosas aos adolescentes, pode influenciar nas escolhas de atitudes saudáveis, por meio de seus ensinamentos ${ }^{10}$. Outro estudo conduzido com adolescentes, com afiliação religiosa, mas que não seguem os hábitos propostos por ela, ou que não tem uma religião, identificou que apresentam maior chance de exposição a comportamentos de risco à saúde, como por exemplo, o consumo de bebidas alcoólicas, de drogas ilícitas, tabaco e envolvimento com dois ou mais parceiros sexuais $^{11}$.

Assim, percebe-se que possuir religião torna-se um fator importante para o estilo de vida saudável de adolescentes. Portanto, o presente estudo teve o objetivo de estimar a prevalência e fatores sociodemográficos e comportamentais associados à afiliação religiosa em escolares do ensino médio no município de Jequié, Bahia, Brasil.

\section{Metodologia}

Trata-se de estudo epidemiológico, transversal, de base escolar, com adolescentes matriculados em escolas públicas de ensino médio do município de Jequié-BA. Este estudo foi aprovado pelo Comitê de Ética em Pesquisa da Universidade Estadual do Sudoeste da Bahia (parecer no 212/2011).

A amostra foi aleatória simples, com substituição do elemento amostral não identificado na escola no dia que foi entregue o convite para participação no estudo. Inicialmente, obteve-se no 13 Núcleo Regional de Educação uma lista com o nome do escolar, data de nascimento, série, turno, nome e endereço das escolas públicas estaduais do ensino médio do município. Em seguida, identificaram-se os escolares com idade de 14 a 19 anos $(n=4.355)$ que, na sequência, foram numerados em ordem crescente (1 a 4.355).

Para o cálculo amostral adotou-se prevalência de inatividade física de $65,7 \% 13$, nível de confiança de $95 \%$ e grau de precisão de $3 \%$, totalizando 788 escolares, aos quais foram acrescidos $10 \%$, prevendo-se as possíveis perdas e recusas, o que elevou a amostra para 867 escolares. A seguir, calculou-se o intervalo amostral $(n=5)$ e sorteou-se a primeira pessoa dentro do intervalo, sendo em seguida a este acrescido o valor do intervalo amostral, com o intuito de identificar os demais escolares participantes do estudo.

De posse do nome do escolar sorteado, os pesquisadores se dirigiram à unidade de ensino para convidá-lo a participar do estudo. Aos adolescentes com idade inferior a 18 anos que aceitaram o convite, foi entregue cópia do Termo de Consentimento Livre e Esclarecido (TCLE) para 
que o seu responsável autorizasse sua participação. Os escolares sorteados para compor a amostra que não se encontravam na sala de aula ou que se recusavam a participar do estudo foram substituídos na lista pelo primeiro nome acima do seu. Tal estudo foi realizado por meio de questionário estruturado, validado para adolescentes ${ }^{14}$.

O instrumento utilizado para a entrevista face a face foi dividido em cinco blocos: perfil sociodemográfico do adolescente e da família; hábitos alimentares (consumo de frituras, doces, frutas, verduras e legumes); nível de atividade física; consumo de tabaco e consumo de álcool. Antes da coleta, houve capacitação para a equipe, com o objetivo de padronizar 0 uso dos instrumentos utilizados na pesquisa. A coleta de dados foi realizada em dia e hora previamente agendados com a direção das escolas, entre os meses de julho a setembro de 2012. Os escolares sorteados que entregaram o TCLE devidamente assinado foram convidados a se dirigir à sala disponibilizada pela direção da escola, onde respondiam ao questionário.

Os critérios de inclusão dos sujeitos no estudo foram: possuir idade entre 14 a 19 anos; estudar em escola pública que oferte ensino médio nas áreas urbana e rural do município de Jequié; estar regularmente matriculado; estar presente na sala de aula no momento da entrega do TCLE e coleta de dados; concordar em participar voluntariamente do estudo.

Foi classificada como variável independente: afiliação religiosa (tem religião; não tem religião). As variáveis dependentes foram: grupo etário (14 a 16 anos; 17 a 19 anos), sexo (masculino e feminino); escolaridade da mãe (Ensino Fundamental e Ensino Médio, ou Superior); com quem mora (com os pais, outros); classe econômica (mais favorecida, A e B; menos favorecida, C, D e E); nível de atividade física (ativos fisicamente - com demanda energética superior a $37 \mathrm{kcal} / \mathrm{kg} / \mathrm{dia}$; inativos fisicamente com demanda energética menor ou igual $36,9 \mathrm{kcal} / \mathrm{kg} / \mathrm{dia}$ ); e hábitos alimentares, consumo semanal adequado ou inadequado de: frituras, doces e refrigerantes, frutas e sucos naturais, verduras e legumes. Foi considerado consumo inadequado de frituras e doces: consumo $\geq 3$ a 4 vezes por semana; e inadequado de frutas e verduras: consumo $<3$ a 4 vezes por semana.
Os dados foram tabulados em duplicata no programa Epi DATA, e após correções foram analisados no programa SPSS versão 21.0. Inicialmente realizou-se análise descritiva das variáveis, e em seguida análises bruta e ajustada por meio de regressão logística binária com a modelagem Backward (para trás condicional). Permaneceram no modelo final as variáveis que apresentassem $p<0,20$. O nível de significância adotado foi $p<0,05$. Os dados foram discutidos com base na literatura recente.

\section{Resultados}

Participaram do estudo 840 escolares, com média de idade de 16,59 anos (mínima=14; máxima=19; DP $\pm 1,363$ ). Houve uma maior percentual de adolescentes: do sexo feminino (60\%); da maior faixa etária de 17 a 19 anos (51\%); que moram pelo menos com um dos pais $(78,2 \%)$; de classe econômica menos favorecida $(75,2 \%)$; que apresentam consumo adequado de frituras $(63,9 \%)$, frutas $(73,3 \%)$ e verduras $(66,2 \%)$, e inadequado de doces (59\%); ativos fisicamente $(55,7 \%)$; filhos de mães com escolaridade correspondente ao ensino fundamental (50\%); e com alguma afiliação religiosa $(84,6 \%)$ (Tabela 1$)$.

Conforme observado na Tabela 2, houve associação da afiliação religiosa com a variável sexo $(p=0,00)$ nas análises brutas e ajustadas. As adolescentes tiveram 2,28 mais chances de pertencer a uma religião dos que os seus pares do sexo masculino.

Ao considerar as análises brutas e ajustadas (Tabela 3), observou-se que adolescentes do sexo masculino, e da faixa etária de 17 a 19 anos apresentaram menor chance de possuir afiliação religiosa, quando comparados a seus pares $(p=0,01 ; p=0,02)$. Aqueles do sexo masculino com mães que possuíam como nível de escolaridade o ensino médio ou superior tiveram duas vezes mais chances de possuir afiliação religiosa do que aqueles com mães com menor grau de escolaridade $(p=0,01 ; p=0,02)$.

$\mathrm{Na}$ Tabela 4, percebe-se que não há associação estatisticamente significante entre a afiliação religiosa e as variáveis sociodemográficas e comportamentais nas adolescentes do sexo feminino. 
Tabela 1. Distribuição de frequências das variáveis sociodemográficas, afiliação religiosa, nível de atividade física e hábitos alimentares dos estudantes do ensino médio do município de Jequié, Bahia, Brasil. 2012.

\begin{tabular}{|c|c|c|}
\hline Descrição das variáveis & $\mathbf{N}$ & (\%) \\
\hline \multicolumn{3}{|l|}{ Sexo $(n=827)$} \\
\hline Masculino & 331 & 40,0 \\
\hline Feminino & 496 & 60,0 \\
\hline \multicolumn{3}{|l|}{ Grupo Etário ( $n=817)$} \\
\hline 14 a 16 anos & 400 & 49,0 \\
\hline 17 a 19 anos & 417 & 51,0 \\
\hline \multicolumn{3}{|c|}{$\begin{array}{l}\text { Mora com pelo menos com um dos pais } \\
(n=834)\end{array}$} \\
\hline $\operatorname{Sim}$ & 652 & 78,2 \\
\hline Não & 182 & 21,8 \\
\hline \multicolumn{3}{|l|}{ Classe Econômica $(n=838)$} \\
\hline Mais favorável (A ou B) & 208 & 24,8 \\
\hline Menos favorável (C,D ou E) & 630 & 75,2 \\
\hline \multicolumn{3}{|l|}{ Escolaridade da mãe ( $n=826)$} \\
\hline Ensino fundamental & 413 & 50,0 \\
\hline Ensino médio ou superior & 413 & 50,0 \\
\hline \multicolumn{3}{|l|}{ Consumo de frituras $(n=830)$} \\
\hline Adequado & 530 & 63,9 \\
\hline Inadequado & 300 & 36,1 \\
\hline \multicolumn{3}{|l|}{ Consumo de doces $(n=831)$} \\
\hline Adequado & 341 & 41,0 \\
\hline Inadequado & 490 & 59,0 \\
\hline \multicolumn{3}{|l|}{ Consumo de Frutas $(n=832)$} \\
\hline Adequado & 610 & 73,3 \\
\hline Inadequado & 222 & 26,7 \\
\hline \multicolumn{3}{|l|}{ Consumo de verduras $(n=826)$} \\
\hline Adequado & 547 & 66,2 \\
\hline Inadequado & 279 & 33,8 \\
\hline \multicolumn{3}{|c|}{ Nível de Atividade Física $(\mathrm{n}=805$ ) } \\
\hline Ativo fisicamente & 357 & 44,3 \\
\hline Inativo fisicamente & 448 & 55,7 \\
\hline \multicolumn{3}{|l|}{ Afiliação Religiosa ( $n=840$ ) } \\
\hline Com Religião & 711 & 84,6 \\
\hline Sem Religião & 129 & 15,4 \\
\hline
\end{tabular}

Tabela 2. Associação entre afiliação religiosa, aspectos sociodemográficos, práticas de atividades físicas e hábitos alimentares dos estudantes (ambos os sexos) do ensino médio do município de Jequié, Bahia, Brasil, 2012.

\begin{tabular}{|c|c|c|c|c|c|}
\hline \multirow{2}{*}{$\begin{array}{l}\text { Variáveis: } \\
\text { sociodemográficas, } \\
\text { atividade física e } \\
\text { hábitos alimentares }\end{array}$} & \multicolumn{5}{|c|}{ Afiliação Religiosa } \\
\hline & $\begin{array}{c}\text { Prevalência } \\
\text { de adolescentes } \\
\text { com afiliação } \\
\text { religiosa }\end{array}$ & OR Bruta (IC95\%) & $\mathbf{p}$ & $\begin{array}{l}\text { OR Ajustada } \\
\text { (IC95\%) }^{1}\end{array}$ & $\mathbf{p}$ \\
\hline Sexo & & & 0,00 & & 0,00 \\
\hline Masculino & 77,9 & 1 & & 1 & \\
\hline Feminino & 88,7 & $2,22(1,52-3,25)$ & & $2,28(1,52-3,40)$ & \\
\hline Grupo Etário & & & 0,11 & & 0,28 \\
\hline 14 a 16 anos & 86,5 & 1 & & 1 & \\
\hline 17 a 19 anos & 82,5 & $0,73(0,50-1,08)$ & & $0,80(0,53-1,20)$ & \\
\hline $\begin{array}{l}\text { Mora com pelo } \\
\text { menos um dos pais }\end{array}$ & & & 0,84 & & 0,82 \\
\hline Sim & 84,7 & 1 & & 1 & \\
\hline Não & 84,1 & $0,84(0,61-1,50)$ & & $0,95(0,58-1,55)$ & \\
\hline Classe Econômica & & & 0,38 & & 0,36 \\
\hline $\begin{array}{l}\text { Mais favorecido (A } \\
\text { ou B) }\end{array}$ & 82,7 & 1 & & 1 & \\
\hline $\begin{array}{l}\text { Menos favorecido } \\
(C, D \text { ou } E)\end{array}$ & 85,2 & $1,21(0,79-1,84)$ & & $1,24(0,80-1,98)$ & \\
\hline Escolaridade da & & & 0,12 & & 0,05 \\
\hline & & & & & Conti \\
\hline
\end{tabular}




\begin{tabular}{|c|c|c|c|c|c|}
\hline mãe & & & & & \\
\hline $\begin{array}{l}\text { Ensino } \\
\text { fundamental }\end{array}$ & 82,8 & 1 & & 1 & \\
\hline $\begin{array}{l}\text { Ensino médio ou } \\
\text { superior }\end{array}$ & 86,7 & $1,35(0,92-1,98)$ & & $1,50(1,00-2,25)$ & \\
\hline Consumo de frituras & & & 0,65 & & 0,98 \\
\hline Adequado & 84,2 & 1 & & 1 & \\
\hline Inadequado & 85,3 & $1,10(0,74-1,63)$ & & $0,99(0,64-1,55)$ & \\
\hline Consumo de doces & & & 0,14 & & 0,51 \\
\hline Adequado & 82,4 & 1 & & 1 & \\
\hline Inadequado & 86,1 & $1,32(0,91-1,93)$ & & $1,15(0,76-1,72)$ & \\
\hline Consumo de Frutas & & & 0,93 & & 0,60 \\
\hline Adequado & 84,4 & 1 & & 1 & \\
\hline Inadequado & 84,7 & $1,02(0,67-1,56)$ & & $1,13(0,71-1,79)$ & \\
\hline $\begin{array}{l}\text { Consumo de } \\
\text { verduras }\end{array}$ & & & 0,80 & & 0,93 \\
\hline Adequado & 84,3 & 1 & & 1 & \\
\hline Inadequado & 84,9 & $1,05(0,70-1,57)$ & & $1,02(0,65-1,61)$ & \\
\hline $\begin{array}{l}\text { Nível de Atividade } \\
\text { Física }\end{array}$ & & & 0,05 & & 0,55 \\
\hline Ativo fisicamente & 81,5 & 1 & & 1 & \\
\hline $\begin{array}{l}\text { Inativo } \\
\text { fisicamente }\end{array}$ & 86,6 & $1,47(1,00-2,15)$ & & $1,14(0,74-1,73)$ & \\
\hline
\end{tabular}

${ }^{1}$ Análise multivariável por meio de regressão logística binária, ajustada pelas seguintes variáveis: sexo, grupo etário, escolaridade da mãe, moradia com os pais, classe econômica, consumo de frituras, consumo de doces e refrigerantes, consumo de frutas ou sucos naturais de fruta, consumo de verduras e níveis de atividade física, método de seleção adotado: Backward Conditional.

IC95\% (Intervalo de confiança = 95\%).

Tabela 3. Associação entre afiliação religiosa, aspectos sociodemográficos, práticas de atividades físicas e hábitos alimentares dos estudantes (sexo masculino) do ensino médio do município de Jequié, Bahia, Brasil. 2012

\begin{tabular}{|c|c|c|c|c|c|}
\hline \multirow[b]{2}{*}{$\begin{array}{l}\text { Variáveis: sociodemográficas, } \\
\text { atividade física e hábitos } \\
\text { alimentares }\end{array}$} & \multicolumn{5}{|c|}{ Afiliação Religiosa } \\
\hline & $\begin{array}{c}\text { Prevalência } \\
\text { de adolescentes com } \\
\text { afiliação religiosa }\end{array}$ & $\begin{array}{l}\text { OR Bruta } \\
\text { (IC95\%) }\end{array}$ & $\mathbf{p}$ & $\begin{array}{l}\text { OR Ajustada } \\
\text { (IC95\%) }^{1}\end{array}$ & $\mathbf{p}$ \\
\hline Grupo Etário & & & 0,01 & & 0,02 \\
\hline 14 a 16 anos & 83,8 & 1 & & 1 & \\
\hline 17 a 19 anos & 71,6 & $\begin{array}{c}0,49(0,28- \\
0,84)\end{array}$ & & $\begin{array}{c}0,52(0,29- \\
0,92)\end{array}$ & \\
\hline $\begin{array}{l}\text { Mora com pelo menos um } \\
\text { dos pais }\end{array}$ & & & 0,36 & & 0,50 \\
\hline $\operatorname{Sim}$ & 78,7 & 1 & & 1 & \\
\hline Não & 73,3 & $\begin{array}{c}0,74(0,39- \\
1,41)\end{array}$ & & $\begin{array}{c}0,78(0,39- \\
1,59)\end{array}$ & \\
\hline Classe Econômica & & & 0,90 & & 0,43 \\
\hline Mais favorecido (A ou B) & 77,5 & 1 & & 1 & \\
\hline Menos favoreido ( $C, D$ ou $E)$ & 78,1 & $\begin{array}{c}1,04(0,60- \\
1,79)\end{array}$ & & $\begin{array}{c}1,28(0,69- \\
2,36)\end{array}$ & \\
\hline Escolaridade da mãe & & & 0,01 & & 0,02 \\
\hline Ensino fundamental & 71,5 & 1 & & 1 & \\
\hline Ensino médio ou superior & 83,5 & $\begin{array}{l}2,01(1,18- \\
3,44)\end{array}$ & & $\begin{array}{c}1,93(1,10- \\
3,38)\end{array}$ & \\
\hline Consumo de frituras & & & 0,84 & & 0,87 \\
\hline Adequado & 77,6 & 1 & & 1 & \\
\hline Inadequado & 78,5 & $\begin{array}{c}1,06(0,61- \\
1,82)\end{array}$ & & $\begin{array}{c}0,95(0,50- \\
1,81)\end{array}$ & \\
\hline Consumo de doces & & & 0,75 & & 0,79 \\
\hline Adequado & 77,2 & 1 & & 1 & \\
\hline Inadequado & 78,7 & $\begin{array}{c}1,09(0,64- \\
1,84)\end{array}$ & & $\begin{array}{c}0,92(0,52- \\
1,65)\end{array}$ & \\
\hline Consumo de Frutas & & & 0,52 & & 0,40 \\
\hline Adequado & 76,9 & 1 & & 1 & \\
\hline Inadequado & 80,2 & $1,22(0,66-$ & & $1,34(0,68-$ & \\
\hline
\end{tabular}


2,25)

\section{Consumo de verduras}

Adequado

Inadequado

\section{Nível de Atividade Física}

Ativo fisicamente

Inativo fisicamente

$\begin{array}{cc}77,8 & 1 \\ 77,2 & 0,96(0,60- \\ & 1,66) \\ 76,3 & 1 \\ 80,3 & 1,27(0,73- \\ & 2,21)\end{array}$
0,89
0,40

2,61)

$$
1
$$

$0,89(0,48-$

$1,66)$

0,80

0,72$$
80
$$

1

$1,08(0,60-$

${ }^{1}$ Análise multivariável por meio de regressão logística binária, ajustada pelas seguintes variáveis: grupo etário, escolaridade da mãe, moradia com os pais, classe econômica, consumo de frituras, consumo de doces e refrigerantes, consumo de frutas ou sucos naturais de fruta, consumo de verduras e níveis de atividade física, método de seleção adotado: Backward Conditional. IC95\% (Intervalo de confiança $=95 \%$.

Tabela 4. Associação entre afiliação religiosa, aspectos sociodemográficos, práticas de atividades físicas e hábitos alimentares dos estudantes (sexo feminino) do ensino médio do município de Jequié, Bahia, Brasil. 2012.

\begin{tabular}{|c|c|c|c|c|c|}
\hline \multirow{2}{*}{$\begin{array}{c}\text { Variáveis: } \\
\text { sociodemográficas, } \\
\text { atividade física e hábitos } \\
\text { alimentares }\end{array}$} & \multicolumn{5}{|c|}{ Afiliação Religiosa } \\
\hline & $\begin{array}{c}\text { Prevalência } \\
\text { de adolescentes com } \\
\text { afiliação religiosa }\end{array}$ & $\begin{array}{l}\text { OR Bruta } \\
\text { (IC95\%) }\end{array}$ & p & $\begin{array}{l}\text { OR Ajustada } \\
\text { (IC95\%) }^{1}\end{array}$ & p \\
\hline Grupo Etário & & & 0,54 & & 0,42 \\
\hline 14 a 16 anos & 87,9 & 1 & & 1 & \\
\hline 17 a 19 anos & 89,7 & $\begin{array}{c}1,19 \\
(0,68-2,10)\end{array}$ & & $\begin{array}{c}1,27 \\
(0,71-2,28)\end{array}$ & \\
\hline $\begin{array}{l}\text { Mora com pelo menos um } \\
\text { dos pais }\end{array}$ & & & 0,89 & & 0,66 \\
\hline Sim & 88,5 & 1 & & 1 & \\
\hline Não & 89,0 & $\begin{array}{c}1,05(0,54- \\
2,02)\end{array}$ & & $1,17(0,57-2,39)$ & \\
\hline Classe Econômica & & & 0,93 & & 0,83 \\
\hline Mais favorecido (A ou B) & 88,4 & 1 & & 1 & \\
\hline Menos favoreido (C,D ou E) & 88,8 & $\begin{array}{c}1,03(0,51- \\
2,08)\end{array}$ & & $1,08(0,52-2,24)$ & \\
\hline Escolaridade da mãe & & & 0,80 & & 0,89 \\
\hline Ensino fundamental & 88,2 & 1 & & 1 & \\
\hline Ensino médio ou superior & 88,9 & $\begin{array}{c}1,08(0,61- \\
1,89)\end{array}$ & & $1,05(0,56-1,97)$ & \\
\hline Consumo de frituras & & & 0,56 & & 0,75 \\
\hline Adequado & 88,0 & 1 & & 1 & \\
\hline Inadequado & 89,7 & $\begin{array}{c}1,19(0,66- \\
2,16)\end{array}$ & & $1,11(0,58-2,09)$ & \\
\hline Consumo de doces & & & 0,15 & & 0,17 \\
\hline Adequado & 86,0 & 1 & & 1 & \\
\hline Inadequado & 90,2 & $\begin{array}{c}1,50(0,86- \\
2,63)\end{array}$ & & $1,49(0,84-2,65)$ & \\
\hline Consumo de Frutas & & & 0,61 & & 0,59 \\
\hline Adequado & 89,0 & 1 & & 1 & \\
\hline Inadequado & 87,4 & $\begin{array}{c}0,85(0,46- \\
1,57)\end{array}$ & & $0,83(0,42-1,64)$ & \\
\hline Consumo de verduras & & & 0,57 & & 0,49 \\
\hline Adequado & 88,1 & 1 & & 1 & \\
\hline Inadequado & 89,9 & $\begin{array}{c}1,19(0,64- \\
2,21)\end{array}$ & & $1,25(0,66-2,36)$ & \\
\hline Nível de Atividade Física & & & 0,66 & & 0,59 \\
\hline Ativo fisicamente & 87,3 & 1 & & 1 & \\
\hline Inativo fisicamente & 88,7 & $\begin{array}{c}1,14(0,64- \\
2,04)\end{array}$ & & $1,18(0,64-2,19)$ & \\
\hline
\end{tabular}

${ }^{1}$ Análise multivariável por meio de regressão logística binária, ajustada pelas seguintes variáveis: grupo etário, escolaridade da mãe, moradia com os pais, classe econômica, consumo de frituras, consumo de doces e refrigerantes, consumo de frutas ou sucos naturais de fruta, consumo de verduras e níveis de atividade física, método de seleção adotado: Backward Conditional. IC95\% (Intervalo de confiança = 95\%). 


\section{Discussão}

Houve predominância de escolares com alta prevalência de afiliação religiosa, majoritariamente do sexo feminino e com idade de 17 a 19 anos. Estudo com escopo similar encontrou valor de $86,5 \%$ para a prevalência de afiliação religiosa em adolescentes, dos quais $74,4 \%$ eram praticantes, $59,7 \%$ eram do sexo feminino, 51,6\% possuíam idade de 17 a 19 anos, e eram ativos fisicamente ${ }^{11}$.

As adolescentes do presente estudo apresentaram mais chances de pertencerem a uma religião do que os seus pares do sexo oposto. Tal fato também foi visto em outro estudo, realizado com adolescentes brasileiros no qual foram observadas médias de religiosidade mais alta entre adolescentes do sexo feminino ${ }^{15}$. Pertencer a uma religião, e ter religiosidade são condições distintas. Entretanto, percebe-se que as adolescentes do sexo feminino se dedicam mais a alguma religião, e quando existir mudanças de hábitos em decorrência disso, isso pode refletir diretamente em seu estilo de vida.

Em relação à idade, no presente estudo, os escolares do sexo masculino com maior faixa etária tiveram menores chances de possuir afiliação religiosa quando comparados aos mais novos. Entretanto, ao considerar que, para ter a religiosidade a pessoa tem que possuir uma religião, nota-se os resultados de um estudo sobre a religiosidade de adolescentes como divergente do estudo atual, pois, percebeu-se apontou os adolescentes na faixa etária de 17 a 19 anos como aqueles com maior média de religiosidade entre os grupos de várias religiões estudadas, com diferenças significativas ${ }^{15}$. Tal fato pode acontecer devido a maior autonomia dos adolescentes com maior faixa etária em optar por participar de ações em instituições religiosas com outros adolescentes da mesma faixa etária.

Os adolescentes do sexo masculino que relataram possuir mães com maior nível de escolaridade (ensino médio ou superior) tiveram mais chances de possuir afiliação religiosa do que aqueles com mães que apresentaram menor grau de escolaridade. Apesar de não ser encontrada relação da escolaridade da mãe dos adolescentes com a afiliação religiosa, em um estudo com adultos brasileiros foi encontrada associação desta variável com comportamentos não saudáveis, como por exemplo, mães com baixa escolaridade e 0 baixo consumo, pelos adolescentes, de frutas e hortaliças e inatividade física no lazer, dentre outros comportamentos ${ }^{16}$.

Estudo com adolescentes mostrou que filhos de mães com maior escolaridade podem apresentar maiores níveis de atividade física de lazer $^{17}$. Desta forma, acredita-se que mães com maior nível de escolaridade podem prover melhores orientações aos filhos em temáticas relevantes para seu bem-estar e contribuir em suas escolhas, as quais poderão refletir em seus comportamentos, estilo de vida ou religiosidade.

$\mathrm{Na}$ amostra pesquisada, as variáveis níveis de atividade física e hábitos alimentares não apresentaram associação significativa com o desfecho estudado. Em relação ao nível de atividade física e afiliação e a prática religiosa existe estudo que corrobora com estes resultados ${ }^{11}$. Entretanto, vale salientar que ao se estudar os fatores associados à afiliação religiosa, sobretudo, fatores relacionados ao estilo de vida, as variáveis em questão são de fundamental importância para a compreensão deste conjunto de hábitos dos adolescentes. Pois, na a manutenção de um estilo de vida saudável devese considerar uma vida fisicamente ativa, dentro dos níveis recomendados (150 a 300 minutos por semana de atividade física moderada a vigorosa) e hábitos alimentares adequados (por exemplo; baixo consumo de doces, salgados, açúcares, gorduras, dentre outros; e elevado consumo de frutas e verduras), evitando, assim, riscos à saúde ${ }^{8}$

O presente estudo apresenta como limitação o fato de não considerar em sua amostra os adolescentes que não frequentam as unidades escolares pesquisadas, não contemplando parte desta população no município estudado. Outra limitação refere-se ao fato de ser um estudo transversal, o que não permite estabelecer relações consistentes de causa e efeito. Entretanto, pode-se considerar que o estudo apresenta uma amostra significativa, dentro de uma população que carece de uma atenção em estudos científicos, por apresentar características próprias de seu local de pesquisa.

\section{Conclusão}

A prevalência de afiliação religiosa em adolescentes matriculados em escolas públicas estaduais no município de Jequié-BA é alta. Foram fatores associados o sexo, grupo etário e escolaridade da mãe. Não foi verificada associação entre o desfecho e práticas de atividades físicas e hábitos alimentares. Adolescentes escolares do sexo feminino, com idade de 17 a 19 anos, om mães com maior nível de escolaridade apresentaram mais chances de pertencer a uma religião. Desta forma, ao 
considerar a importância da afiliação religiosa na mudança de hábito dos adolescentes, sugere-se aprofundar o estudo das relações deste desfecho com as variáveis de estilo de vida.

\section{Referências}

1. Cabistany LD, Rombaldi AJ. Associação entre prática religiosa e estilo de vida saudável em escolares de Pelotas, RS. ABCS Health Sci. [internet] 2014 Mai [acesso em 2019 Jul 1]; 39(2):64-70. Disponível em: DOI: http://dx.doi.org/10.7322/abcshs.v39i2.624.

2. Valim GG, Simionato P, Gascon MRP. O consumo de álcool na adolescência: uma revisão literária. Adolesc Saude. [internet] 2017 Out-Dez [acesso em 2019 Set 16]; 14(4):184-94. Disponível em: http://www.adolescenciaesaude.com/detalhe_ar tigo. asp? id $=698$.

3. Ferrari CKB, Carneiro SS, Rocha EM, Santos ALV. Sedentarismo, estilo de vida e saúde em adolescentes de um município da amazônia legal. REVISTA INSPIRAR [internet] 2017 Jul [acesso em 2019 Ago 14]; 14(3): 28-33. Disponível em: https://www.inspirar.com.br/revista/sedentaris mo-estilo-de-vida-e-saude-em-adolescentes-deum-municipio-da-amazonia-legal/.

4. Menezes AHR, Dalmas JC, Scarinci IC, Maciel SM. Fatores associados ao uso regular de cigarros por adolescentes estudantes de escolas públicas de Londrina, Paraná, Brasil. Cad Saúde Pública. [internet] 2014 Abr [acesso em 2019 Jul 28]; 30(4):774-84. Disponível em: http://www.scielo.br/pdf/csp/v30n4/0102-311Xcsp-30-4-0774.pdf.

5. Vargas LS, Lucchese $R$, Silva AC, Guimarães RA, Vera I, Castro PA. Determinantes para o consumo de tabaco por estudantes. Rev Saude Publica. 2017;51:36.

6. Baldissera G, Silva SP, Zaneti ICBB, Hagen MK, Magalhães CR. Práticas e hábitos alimentares de crianças e adolescentes: a relação entre os aspectos socioculturais e midiáticos. Cad pedagógico. [internet] 2015 [acesso em 2019 Jul 30]; 12(1):289-300. Disponível em: http://www.univates.br/revistas/index.php/cadp ed/article/view/952/940.

7. Valentim EA, Almeida CCB, Taconeli CA, Osório MM, Schmidt ST. Fatores associados à adesão à alimentação escolar por adolescentes de escolas públicas estaduais de Colombo, Paraná, Brasil. Cad Saúde Pública. [internet] 2017 Out [acesso em 2019 Ago 29]; 33(10):e00061016. Disponível em: http://www.scielo.br/scielo.php?pid=S0102-
$311 \times 2017001005002 \&$ script=sci_abstract $\&$ tlng $=p$ t.

8. Nahas MV. Atividade física, saúde e qualidade de vida: conceitos e sugestões para um estilo de vida ativo. 7a ed. Londrina: Midiograf; 2017.

9. Regis MFR, Oliveira LMFT, Santos ARM, Leonidio ACR, Diniz PRB, Freitas CMSM. Estilo de vida urbano versus rural em adolescentes: associações entre meio-ambiente, níveis de atividade física e comportamento sedentário. Einstein. [internet] 2016 out [acesso em 2019 Ago 30]; 14(4):461-7. Disponível em: http://www.scielo.br/pdf/eins/v14n4/pt_16794508-eins-14-04-0461.pdf.

10. Santos ARM, Silva EAPC, Silva PPC, Cartaxo HGO, Freitas CMSM. Estilo de vida na adolescência: o envolvimento religioso atuando nos comportamentos de risco à saúde. Pensar prát. [internet] 2014 Jan-Mar [acesso em 2019 Ago 15]; 17(1):01-294. Disponível em: https://pesquisa.bvsalud.org/portal/resource/pt/ biblio-869237.

11. Santos ARM, Oliveira LMFT, Farias JC Jr, Silva PPC, Silva EAPC, Freitas CMSM. Associação entre prática religiosa e comportamentos de risco à saúde em adolescentes de Pernambuco, Brasil. Rev Bras Ativ Fís Saúde. [internet] 2015 Out [acesso em 2019 Jul 15]; 20(3):284-96. Disponível http://rbafs.org.br/RBAFS/article/view/5046.

12. Bezerra MRE, Lyra MJ, Santos MAM, Colares V, Menezes VA. Fatores de Risco Modificáveis para Doenças Crônicas não Transmissíveis em Adolescentes: Revisão Integrativa. Adolesc Saude. [internet] 2018 AbrJun [acesso em 2019 Dez 20]; 15(2):113-20. Disponível em: http://www.adolescenciaesaude.com/detalhe_ar tigo.asp?id=726.

13. Farias JC Jr, Lopes AS. Comportamentos de risco relacionados à saúde em adolescentes. Rev Bras Ci e Mov. [internet] 2004 Jan-Mar [acesso em 2019 Dez 18]; 12(1):7-12. Disponível em:

https://portalrevistas.ucb.br/index.php/RBCM/ar ticle/view/535.

14. Farias JC Jr. Estilo de vida de adolescentes do município de Florianópolis, Santa Catarina, Brasil [dissertação]. Florianópolis: Universidade Federal de Santa Catarina, Centro de Desportos; 2002. 135 p.

15. Jahn GM, Dell'Aglio DD. A Religiosidade em Adolescentes Brasileiros. Rev Psicol IMED. [internet] 2017 Jan-Jun [acesso em 2019 Nov 25]; 9(1): 38-54. Disponível em: http://pepsic.bvsalud.org/scielo.php?script=sci_a 
rttext\&pid=S2175-50272017000100004.

16. Faleiro JC, Giatti L, Barreto SM, Camelo LV, Griep RH, Guimarães JMN, et al. Posição socioeconômica no curso de vida e comportamentos de risco relacionados à saúde: ELSA-Brasil. Cad Saúde Pública. [internet] 2017 Abr [acesso em 2019 Nov 10]; 33(3):e00017916. Disponível em: http://www.scielo.br/scielo.php?pid=S0102$311 X 2017000305005 \&$ script $=$ sci_abstract $\&$ tlng $=p$ t.

17. Ferreira RW, Varela $A R$, Monteiro $L Z$, Häfele CA, Santos SJ, Wendt $A$, et al. Desigualdades sociodemográficas na prática de atividade física de lazer e deslocamento ativo para a escola em adolescentes: Pesquisa Nacional de Saúde do Escolar (PeNSE 2009, 2012 e 2015). Cad Saúde Pública. [internet] 2018 Mai [acesso em 2019 Nov 25]; 34(4):e00037917. Disponível em:

www.scielo.br/scielo.php?script=sci_abstract\&pi $\mathrm{d}=\mathrm{S} 0102$

$311 X 2018000405015 \& \operatorname{lng}=p t \& n r m=i s o$.

\section{Endereço para Correspondência}

Universidade do Estado da Bahia (UNEB) Campus XII

Loteamento Ipanema, s/n, Bairro Ipanema, Guanambi (BA)

CEP $46430-000$

e-mail: cbalmeida@uneb.br

Recebido em 15/10/2020

Aprovado em 19/03/2021

Publicado em 31/03/2021 